\title{
SN1987A: the X-ray remnant at age 25 years
}

\author{
David N. Burrows ${ }^{1}$, Sangwook Park ${ }^{2}$, Eveline A. Helder ${ }^{1}$, \\ Daniel Dewey ${ }^{3}$, Richard McCray ${ }^{4}$, Svetozar A. Zhekov ${ }^{5}$, \\ Judith L. Racusin ${ }^{6}$, and Eli Dwek ${ }^{6}$ \\ ${ }^{1}$ Dept. of Astronomy \& Astrophysics, Penn State University, \\ University Park, PA 16802 USA; email: burrows@astro.psu.edu; \\ ${ }^{2} \mathrm{U}$. Texas-Arlington, Arlington, TX, USA; ${ }^{3}$ MIT Kavli Institute, Cambridge, MA, USA \\ ${ }^{4}$ U. Colorado, Boulder, CO, USA; ${ }^{5}$ Space Research and Technology Institute, Sofia, Bulgaria \\ ${ }^{6} \mathrm{NASA} / \mathrm{GSFC}$, Greenbelt, MD, USA
}

\begin{abstract}
SN1987A is the best-studied core-collapse supernova in the sky. We know what the progenitor was, what the circumstellar environment was, and what the explosion looked like over a broad electromagnetic bandpass and in neutrinos. For over a decade, the Chandra X-ray Observatory has been monitoring SN1987A on a regular basis, obtaining resolved images of the developing interaction with the circumstellar material, as well as high resolution grating spectroscopy of the X-ray emission. We highlight the latest results from this campaign and discuss the overall picture of the remnant's structure that emerges from these observations.
\end{abstract}

Keywords. X-rays:individual(SN1987A), stars:supernovae:individual(SN1987A)

\section{Introduction}

Supernova SN1987A is unique among the myriad supernovae discovered in the past century. It was the first naked-eye supernova since 1604 (Kepler). Unlike the Galactic supernova remnants (SNRs), it has an accurately known distance (51.4 $\pm 1.2 \mathrm{kpc}$; Panagia et al. 1999); a known type (Type IIP), and even a known progenitor (Sk -69 202, a B3I blue supergiant). The explosion time (07:36 UT on 23 February 1987) is known precisely due to the detection of neutrinos from the event (Hirata et al. 1987), which also confirmed that it was a core-collapse supernova. Because of its proximity in the Large Magellanic Cloud, it has been observed across the electromagnetic spectrum. It is without a doubt the best-studied supernova to date.

SN1987A was also unique in a number of its characteristics. The fact that the progenitor was a blue supergiant was quite surprising at the time. HST began making high-resolution optical observations shortly after its launch in 1990, discovering the unique triple-ring system that was illuminated by the UV flash from the explosion. Subsequent HST observations found unexpected "hot spots" on the inner ring (Lawrence et al. 2000), and tracked the expansion of the ejecta inside the inner ring (Larsson et al. 2011).

Chandra began making high resolution X-ray images shortly after its launch in 1999 and has been tracking the X-ray behavior ever since, in both high-resolution images and high-resolution spectography. Here we highlight recent results from our Chandra campaign with the Advanced CCD Imaging Spectrometer (ACIS), spanning the years $1999-2011$. 


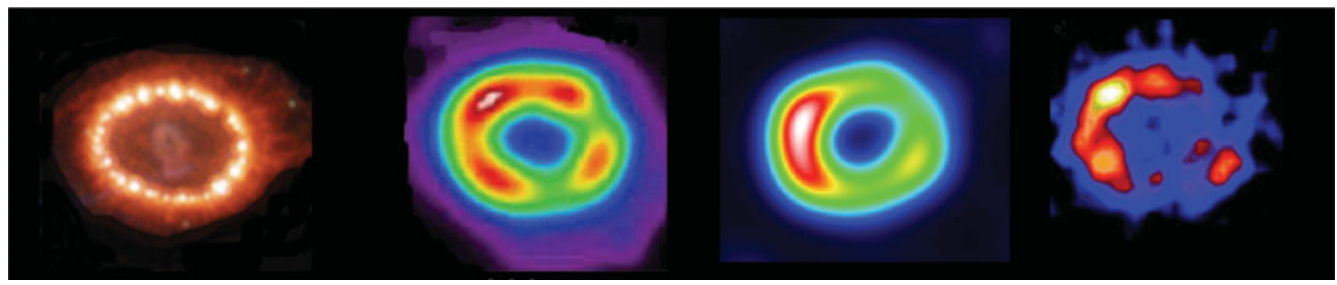

Figure 1. SN1987A ca. 2005. Left to right: HST, Chandra 0.5-2 keV, ATCA $12 \mathrm{~mm}$ (Manchester et al. 2005), Gemini $12 \mu \mathrm{m}$ (Bouchet et al. 2006).

\section{Multiwavelength Overview}

We begin with a very brief multiwavelength overview (Fig. 1). From left to right:

Optical. The inner equatorial ring (ER) begins to develop hot spots ca. 1995, and these have now encircled the entire ring. The ejecta in the center are expanding. After dimming until day $\sim 5000$ due to radioactive decay of ${ }^{44} \mathrm{Ti}$, they are now brightening again as the soft X-rays from the ER have begun to reheat the ejecta (Larsson et al. 2011).

$X$-ray. The X-ray image shows an expanding ring of emission due to interactions of the shock with the HiI region inside of the ER, and with the ER itself. Bright spots in the NE and SE quadrants correspond to optical hot spots \#1 (NE) and \#2-4 (SE).

$\underline{\text { Radio. }}$. The radio image also shows an expanding shell-like structure, but the eastern emission is distributed somewhat differently than the soft X-rays, while the bright spot in the west aligns well with the western bright spot in the X-ray image.

Infrared. The $12 \mu \mathrm{m}$ image corresponds very well to the X-ray image. Spitzer measurements show that the mid-IR flux tracks the soft X-ray flux quite well, providing evidence that the mid-IR component is due to dust heated to $T \sim 180 \mathrm{~K}$ by collisions with electrons in the hot X-ray emitting shocked ER (Dwek et al. 2010). Herschel observations at 100-350 $\mu \mathrm{m}$ suggest that roughly 0.5 solar masses of dust in the ejecta have been heated to $20 \mathrm{~K}$, possibly also by the soft X-rays from the ER (Matsuura et al. 2011).

\section{X-ray Observations}

The Chandra/ACIS instrument has monitored SN 1987A roughly every six months since October 1999. The observations have been taken in several instrument configurations to obtain both high resolution images and high resolution spectra. There is space here only to sketch a few key results; for more details please see papers published between 2000 and 2011 by S. Park, S. Zhekov, D. Dewey, J. Racusin, and references therein.

Images. We have produced deconvolved images at each observational epoch. Details of the technique are given in Burrows et al. (2000). A few images are shown in Figure 2. The emission is dominated by dense gas in the inner ring heated to $\sim 3 \times 10^{7} \mathrm{~K}$ by the forward shock, indicating that SN 1987A is now a supernova remnant.

Light Curve. The Chandra soft X-ray light curve of SN 1987A is shown in Figure 3. The flux increased linearly for over five years, from day 6500 to 8500 , but then flattened out and has been nearly constant for the last 2.5 years (Park et al. 2011). This flattening of the light curve suggests that the shock wave has passed through the peak density of the inner ring and may be about to enter the region outside the visible ring, where it will map out the unknown density profile beyond the ER.

Expansion. The superb angular resolution of Chandra has allowed us to measure the expansion of the X-ray remnant, which is shown in Fig. 4. The expansion rate slowed dramatically around day 6000 to about $1780 \mathrm{~km} / \mathrm{s}$ (Racusin et al. 2009). 

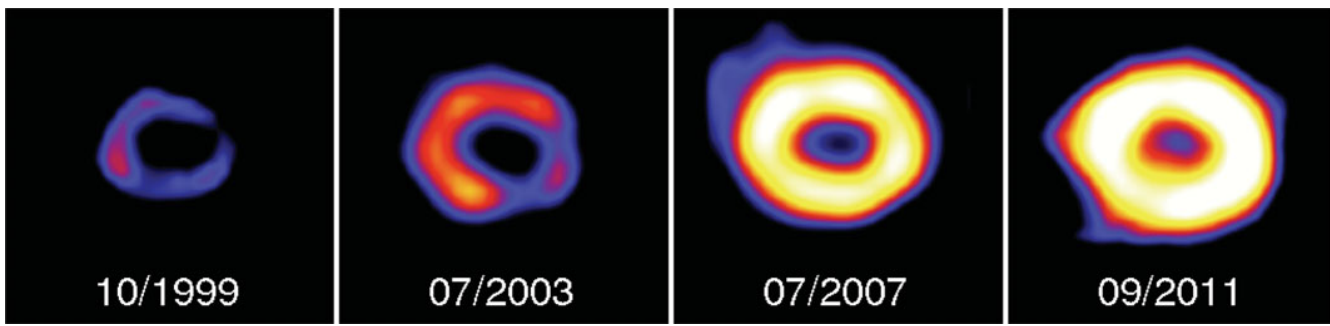

Figure 2. Deconvolved, smoothed images of SN 1987A from $1999-2011$. The color scale is logarithmic. North is up, East is to the left.

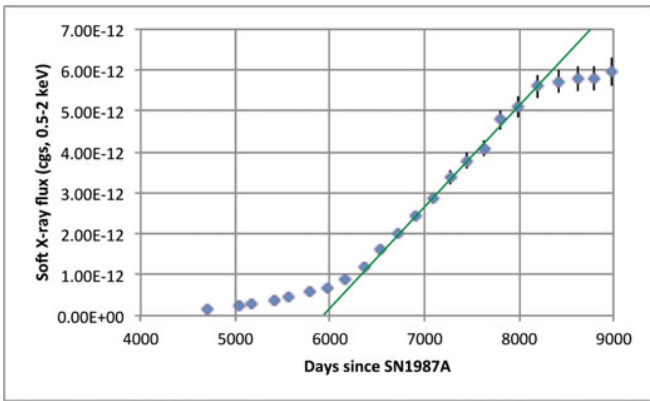

Figure 3. Chandra soft X-ray light curve of SN 1987A.

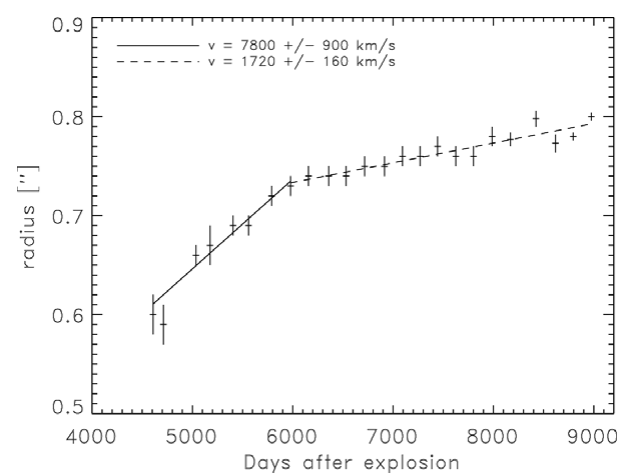

Figure 4. ACIS expansion measurements of SN 1987A.

Compact Remnant. SN 1987A is expected to contain a neutron star buried under optically thick ejecta (Graves et al. 2005), which could emerge from the ejecta at any time as the latter continue to expand and thin out. However, our observations show no evidence to date for a central source, with a limit of $1.5 \times 10^{34} \mathrm{erg} / \mathrm{s}$ on the observed flux.

\section{Physical Model}

The physical model for SN 1987A inferred by Dewey et al. (2012; hereafter D12) is illustrated in Fig. 5. Analysis of the X-ray images and line spectra indicate that the X-ray emission is produced by 3 components: the shocked, clumpy ER (red regions in Fig. 5), which currently dominates the soft X-ray band; the shocked HII regions inside the ER and off the equatorial plane (blue regions in Fig. 5), which produce very broad X-ray line profiles and which dominate the hard X-ray and radio bands; and the reverseshocked outer ejecta (green regions in Fig. 5). Zhekov et al. $(2009,2010)$ fit the in-plane components of this model 
to ACIS light curves and spectra up to 2007. D12 fit this model, including the outof-plane HII regions, to the measured X-ray fluxes and line profiles to determine the parameters of a set of hydro models that reproduce both the spectra and light curves.

\section{The Future of SN1987A}

The Forward Shock. The forward shock is expected to pass beyond the dense inner ring in the near future, when it will map out the circumstellar density profile, acting as a "time machine" to measure the mass loss history of Sk -69 202. D12 show that the future $\mathrm{X}$-ray light curve will differ dramatically, depending on whether or not the ER continues beyond the visible ring to larger radii. The same will apply to the IR light curve, which is closely coupled to the X-ray flux.

The Ejecta. Optical observations show that the bulk of the ejecta is rapidly approaching the inner ring (Larsson et al. 2011), but both optical images and the hydrodynamical simulations of D12 suggest that the reverse shock has not yet entered this material. The early emergence of X-rays from SN 1987A demonstrated that the supernova explosion ejected some of the newly synthesized ${ }^{56}$ Co into the outer envelope of the supernova ejecta (McCray 1993). Eventually, clumps of ${ }^{56}$ Co and other newly synthesized elements in the outer debris will cross the reverse shock, producing dramatic changes in the X-ray spectra. Such events could happen at any time during the next several years. Frequent monitoring of SN 1987A at all wavelengths is required to monitor these expected changes.

The Compact Remnant (?). As the ejecta continue to expand and thin, we expect that the putative neutron star at the center of SN 1987A will become apparent. We have not been able to detect it yet in the Chandra bandpass, but it is possible that NuSTAR, which will be launched in March 2012, will find evidence at higher energies for a pulsar.

The future should continue to deliver exciting new results, as the forward and reverse shocks probe uncharted territory in the CSM and ejecta, respectively. The continued development of the youngest accessible supernova remnant will undoubtedly continue to puzzle and enlighten us for years to come.

\section{References}

Bouchet, P., et al. 2006, ApJ, 650, 212

Burrows, D. N., et al. 2000, ApJ, 543, L149

Dewey, D. \& Dwarkadas, V. V., et al. 2012, ApJ, submitted, arXiv 1111.5314

Dwek, E., et al. 2010, ApJ, 722, 425

Graves, G. J. M., et al. 2005, ApJ, 629, 944

Hirata, K., et al. 1987, Phys. Rev. Lett., 58, 1490

Larsson, J., et al. 2011, Nature, 474, 484

Lawrence, S., S., et al. 2000, ApJ, 537, L123

Manchester, R., et al. 2005, ApJ, 628, L131

Matsuura, M., et al. 2011, Science, 333, 6047

McCray, R. 1993, ARAA, 31, 175

Panagia, N. 1999, in: Y.-H. Chu, N. Suntzeff, J. Hesser, \& D. Bohlender (eds.), New Views of the Magellanic Clouds, Proc. IAU Symposium No. 190, p. 549

Park, S., et al. 2011, ApJ, 733, L35

Racusin, J. L., et al. 2009, ApJ, 703, 1752

Zhekov, S. A., et al. 2009, ApJ, 692, 1190

Zhekov, S. A., et al. 2010, MNRAS, 407, 1157 